\title{
Machine Learning Techniques for Automated Tremor Detection in the Presence of External Stressors
}

\author{
K. M. Vanitha ${ }^{1}$, Viswanath Talasila ${ }^{2}$ \\ ${ }^{1}$ Department of Electronics and Instrumentation Engineering, Ramaiah Institute of Technology, Vidya \\ Soudha, MSR Nagar, MSRIT Post, Bangalore - 560054, India \\ Email:kmvanitha@msrit.edu \\ ${ }^{2}$ Department of Telecommunication Engineering, Ramaiah Institute of Technology, Vidya Soudha, \\ MSR Nagar, MSRIT Post, Bangalore - 560054, India \\ Email: viswanath.talasila@msrit.edu
}

Received: June 18, 2021. Revised: December 10, 2021. Accepted: January 13, 2022. Published: January 14, 2022.

\begin{abstract}
In this study tremor data of 25 subjects (Senile tremor $=5$, Alcohol induced tremor $=9$, Healthy individuals $=11$ ) were collected using a wearable device consisting of five Inertial Measuring Units (IMUs) and an embedded optical sensor. The subjects were made to draw the Archimedes spiral under the influence of external stressors. Features were extracted from measured acceleration data and also from an optical sensor. Using the selected features few supervised machined learning algorithms were explored for automatic classification of tremor. Performance matrix used to evaluate the classifier was accuracy, recall, and precision. It is observed that the algorithms are able to accurately classify healthy, senile tremor and alcohol induced tremor.
\end{abstract}

Keywords- Tremor, Wearable sensing device, Archimedes spiral, Automatic scoring, Machine learning algorithm.

\section{INTRODUCTION}

Research on tremor classification is vast and growing rapidly. Human tremors are assessed by using clinical rating scales and biomechanical sensors. Analysis using spiral drawing is a popular method which is simple, easy to administer [10]. Tracing the spiral is considered as a better approach as it activates the multiple muscles and joint in the arms [10]. Several studies use the automatic scoring system and clinical rating scale in unison to attain a more objective and sophisticated diagnosis and to provide clinical efficacy [56].

\section{A. Background}

Rhythmic oscillatory movement of a body part is defined as tremor and is the most common movement disorder. Tremor is difficult to diagnose and manage [57]. Tremor is a disease which progresses gradually. It is associated with indications such as postural instability, slowness of movement, and rigidity [1-3]. Rigidity can be defined as inflammation and opposition to movement in joints. For most tremor patient's symptoms are observed in the upper extremities of the body. Tremor patients facing problems or slowness when performing daily life tasks such as dressing, writing and using utensils is an indication of slowness of movement. Method for an assessment of slowness of movement usually identified by making the patients perform repetitive and cyclic movements of the upper limbs. Postural instability is seen at a later stage as the ailment progresses.

Tremors are influenced by drugs, physiological and psychological factors. Tremors vary in frequency and amplitude. Categorization of tremor depends on posture, position and the movement. A resting tremor elicits when the body part is still [57]. A postural tremor transpires with sustained posture and kinetic tremor with displacement [57]. Essential tremor, which is a most common tremor, has a cycle rate between four and eight $\mathrm{Hz}$ and occurs in the upper and lower extremities. Parkinson's disease (PD) causes a 4 to $6 \mathrm{~Hz}$ resting tremor in arms and legs. Tremor associated with $\mathrm{PD}$ occurs when the muscles are relaxed and are at rest. Tremor can also be a manifestation of various diseases like Wilson's disease, lesions of the cerebellum and midbrain, peripheral neuropathy, trauma, abuse of alcohol, and conversion disorders [17].

Life of a tremor patient is impacted during the entire duration of the disease. In some patients, tremor reduces in the latestage [18] [19]. This could be attributed to the incongruity between tremor rankings among initial and late-stage tremor subjects. Tremor is a significant cause of social anxiety and mortification that deleteriously affects initial-stage patient's 
sense of safety, and comfort [18] [19]. Louis et al. conducted a survey of 103 tremor patients and summarized that more than one-quarter agreed with experiencing embarrassment [20]. In an independent survey of 100 tremor patients, 36\% expressed an experience of social stigma [21]. Findings from the research emphasize the psychosocial impact on patients with early-stage tremor. Tremor also affects day today life of patients. More than $30 \%$ of people with early-stage tremor expressed tremorrelated difficulty with writing, using a type-writer or computer keyboard, using mobiles, dressing, ingestion, and holding a book [20]. Average age at tremor onset is found to be 55-65 years $[22,23]$, many people are still labouring when they first noticed tremor.

There are various tremor assessment techniques [33, -37]. An effective method for early detection of tremor would be a boon to the disease detecting process. Spiral analysis uses Archimedean spiral to measure motor activity. It is also used to assess the movement disorder symptoms such as tremor, firmness, and slowness of movement [4] [5]. In 2008, Pullman et al. carried out a legitimacy study of spiral analysis for its suitability to motor activity evaluation in Parkinson Disease [6]. Liu et al. measured drug-induced dyskinesia in the arms using spiral templates of a circular and a square shape [7]. Miralles et al. proposed a quantitative method using digitized image of Archimedean spiral [8]. Aly et al. proposed automation for estimation of tremor using a task of tracing a pentagon shaped spiral [9]. There are also other research studies based on spiral analysis and applications developed for mobile platforms for tremor detection [11-15].

Digital drawing tablets, laser-based displacement transducers, video tracking, Infrared cameras, and electromyography (EMG) have been used to evaluate tremors quantitatively [2432]. Accelerometer provides objective measure of tremor frequency and hence is generally accepted as a gold standard [38,39]. Fourier transform is one of the method for determination of tremor frequency and amplitude. Analysis is often done in frequency domain using the methods based on (Fast Fourier Transform (FFT), Power Spectral Density (PSD)) [40, 41]. For example, the tremor frequency is determined as the peak in the PSD. Tremor amplitude can be estimated from the area under PSD peak. Alternatively, there are studies in the literature such as time-domain approaches based on thresholds [41], approaches based on Short Term Fourier Transform (STFT), Empirical Mode Decomposition (EMD) [43], parametric identification methods [42]. Gravity and inertial acceleration was investigated as an alternative to commonly used direct spectral analysis methods of measuring acceleration [44].

Studies were conducted for automatically evaluating the score for gait tasks [46-49],[58], bradykinesia [45, 50], fingertapping task [51,52], and PD tremors [53-55]. Supervised learning and regression models were used to automatically obtain a score for gait and bradykinesia tasks [45-55]. Supervised learning models were used to evaluate the rating scale for the digit-tapping task and Parkinson Disease tremors [51-55].

However, in the literature there have been no studies which focus on effect of external stressors. In previous work [59], presented the design of a smart glove for measuring tremors in real time, while the subjects were tracing an Archimedes Spiral. The study [59] was limited to just two subjects and the acquired measurements were not sufficient for accurate classification. In the present study, a low-cost wearable sensing system is used which shall be worn by the subject such that a sensor is mounted on each finger of the patient. With the help of a laser diode mounted on the index finger, patient shall re-trace the spiral displayed on the screen. Patients are instructed to climb the stairs up and down and then trace the spiral. Statistical features are extracted from accelerometer and spirogram pixel distance signals. These parameter or features are fed as input to machine learning algorithms for automatic classification of tremor. The paper is organized into two subdivisions. In subdivision 2, materials, methods and tremor analysis during a task subject to external stressors have been discussed. In section 3 , we present the experimentation and analysis on tremor subjects and healthy subjects from the test is discussed. Therefore, this paper explains a machine-learning methodology to objectively measure and precisely assess tremor.

\section{MATERIALS AND METHODS}

This research work was carried out at Aluru Primary health centre which is located at Davanagere Taluk, Karnataka, India. The subjects were pre-screened for presence of tremor by a physician and selected for the study. The clinician was instrumental in selection and also supervised the process of data collection. Twenty-five subjects with and without tremor (average age: $59 \pm 10$-years, 7 females, 18 males) participated in this study. All of these subjects (rural and urban) voluntarily participated in the study. Among them fourteen were clinically diagnosed as experiencing tremor.

\section{A. Data Acquisition and Analysis}

A low-cost custom designed wearable sensing system [58] was used for this study to acquire the tremor data. Figure 1 shows the wearable sensing device used for the study and pictures of subjects wearing sensing device. The wearable glove is equipped with five IMUs (MPU6050) which are interfaced to a microcontroller via an Inter Integrated Circuit $\left(\mathrm{I}^{2} \mathrm{C}\right)$ multiplexer. The triple-axis Micro Electro Mechanical Systems (MEMS) accelerometer in MPU6050 can sense up to $\pm 16 \mathrm{~g}$ along the three Cartesian axes, and the gyroscope has sensitivity of $\pm 2000 \mathrm{dps}$ (degrees per second). 


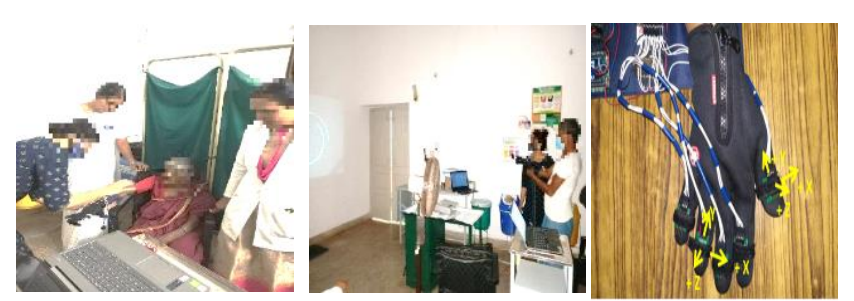

Figure 1. Subjects with wearable sensing device.

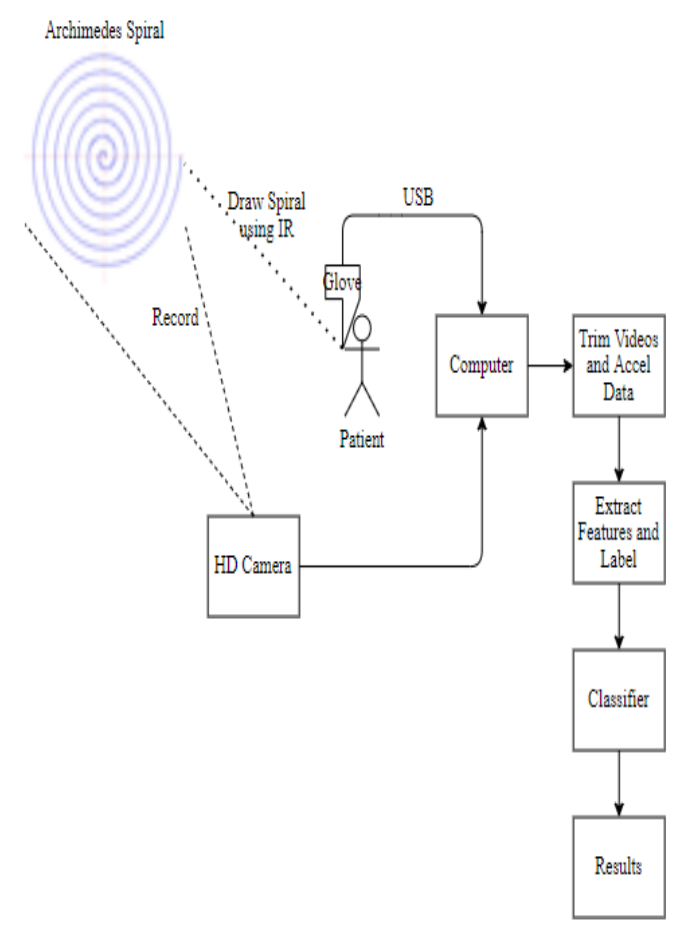

Figure 2. Experimental setup and data processing

The experimental setup used for data acquisition and analysis is as shown in Figure 2. The subjects were asked to retrace an Archimedes spiral projected on the screen under external stressors. Archimedes spiral as in Figure 2, is a locus corresponding to the locations over time of a point moving farther from a reference point. The spiral is projected onto a wall, the subject's glove is embedded with a high intensity LED and IMUs and the subject is asked to trace the projected spiral image while standing at a distance of 4.5 meters and not supported against gravity. The subjects were subjected to external stressors, they were made to climb a flight of stairs multiple times (Subjects run down and up one floor for one time, two time and three times). Immediately after the completion of the activity, they were made to trace the Archimedes spiral while wearing the instrumented glove. Tremor signals were acquired when each subject was tracing the Archimedes spiral which was projected on a screen for 80 seconds. A High Definition (HD) camera simultaneously recorded the spiral tracing for evaluation. In our work all five Inertial Measuring Units (IMU) accelerometer data was recorded at a sampling rate of $100 \mathrm{~Hz}$. Acquired accelerometer data was segmented after eliminating the first $10 \mathrm{~s}$ and the last $10 \mathrm{~s}$ in order to remove any data outliers. Accelerometer data representing acceleration in three cartesian direction is converted into $g$ unit, where $g$ represents acceleration due to gravity $\left(\mathrm{g}=9.81 \mathrm{~m} / \mathrm{s}^{2}\right)$. Then the absolute value of

acceleration, $a_{x y z}(n)$ is calculated as follows

$a_{x y z}(n)=\sqrt{a_{x}^{2}(n)+a_{y}{ }^{2}(n)+a_{y}{ }^{2}(n)} \ldots \ldots(1)$

FFT analysis of the pre-processed data from all five IMUs is performed. FFT plots of Power spectral density (PSD) for two subjects are shown in Figures 4, 5 and rest in Appendix. Figure 3 shows the steps followed to obtain FFT PSD plots. Accelerometer raw data is converted to $1 \mathrm{~g}$ data by dividing raw data values with a scaling factor as specified in the IMU datasheet. Absolute value of acceleration is calculated from $x$, $\mathrm{y}$ and $\mathrm{z}$ axis $1 \mathrm{~g}$ converted data. Absolute value is filtered using Butterworth filter of fifth order with a cut off frequency of 25 Hz. FFT is computed on the filtered data and PSD plots are obtained.

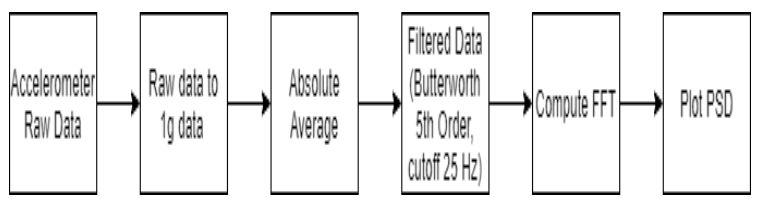

Figure 3. Flow diagram to obtain FFT-PSD plots

The spiral tracing was recorded using a HD camera this was processed offline to calculate Pixel Distance Error (PDE). PDE is the distance between the point on the reference spiral and the corresponding point on the path traced by the subject. Pixel Distance Error (n) = abs (Reference Pixel (n) - Traced Pixel (n)

The traced Archimedes spiral drawn is captured using a camera system, for a total of 14 subjects is shown from Figures 4 and 5 for two subjects and the rest are in Appendix. Figures 4 and 5 shows pixel distance for the collected data. Pixel distance between Archimedes and traced spirals is calculated. As seen from the Figure 4 and 5, it is observed that mean pixel distance for subjects (having clinical tremor) is larger than subjects (who do not have clinical tremor). Mean pixel distance of subjects (having clinical tremor) is far from zero because of non-smooth tracings with sharp edges. Mean pixel distance of subjects (who do not have clinical tremor) is closer to zero because of smooth tracings. Mean, Median, Variance and Standard deviation are computed for the subjects (with and without tremor) and plots are shown in Figures 6, 7, 8 and 9. We also observed that the Alcohol induced tremor subjects have a standard deviation of pixel distance error less than 10 and Senile tremor subject's standard deviation of pixel distance error above 15 . 

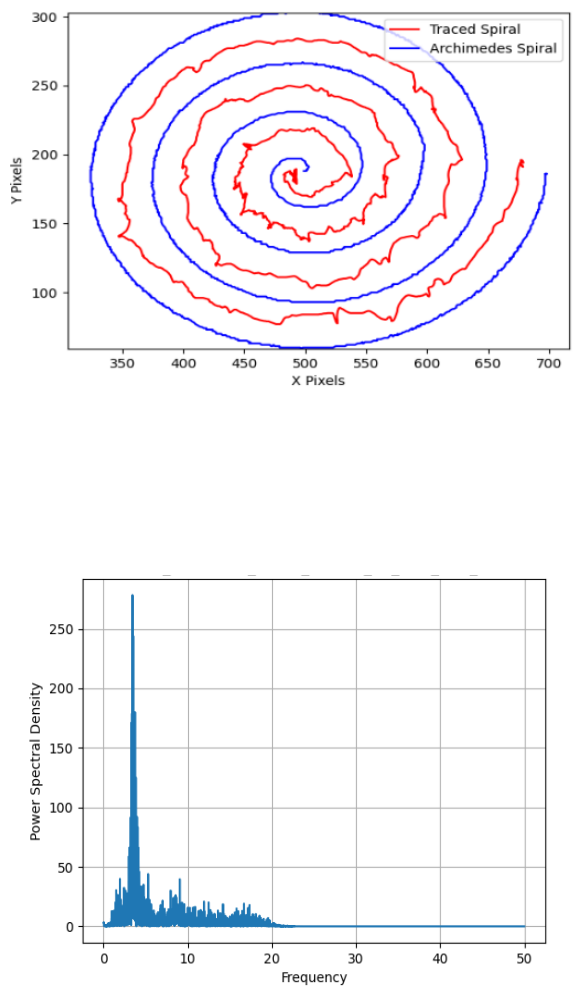

Subject without tremor - tracing Archimedes spiral after climbing flight of stairs Note: Jumps are less pronounced.

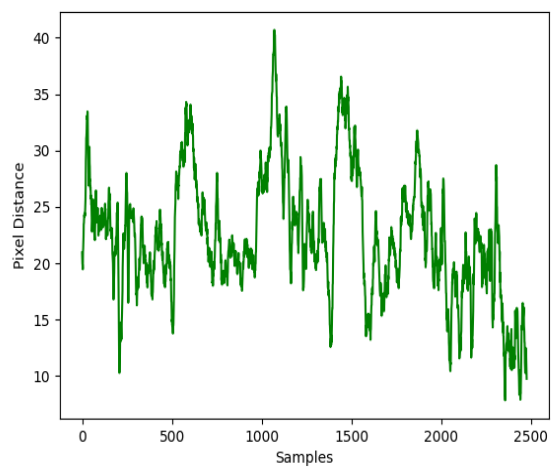

Pixel distance between Archimedes spiral and subject without tremor traced line

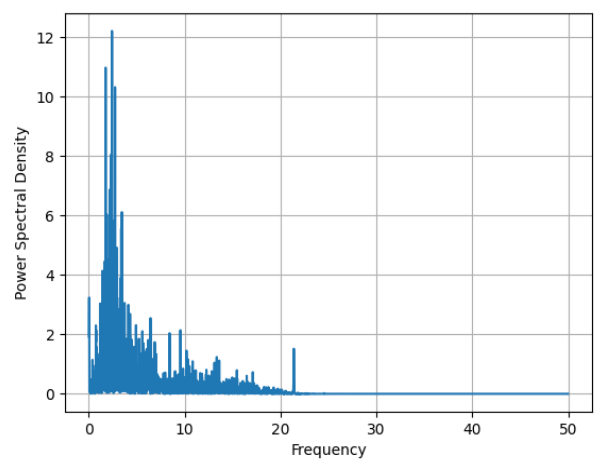

Power spectral density of subject without tremor

Figure 4. Subject without tremor - Age 62

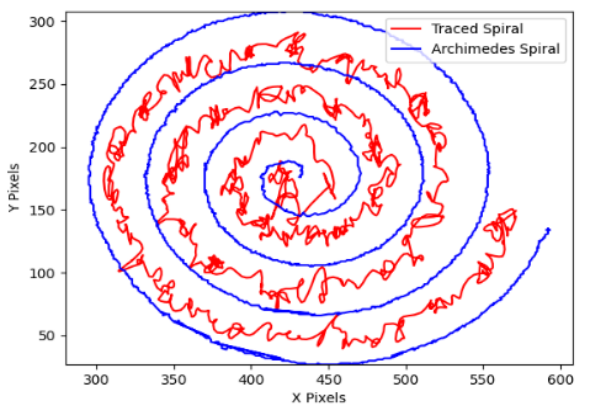

Subject with senile tremor - tracing Archimedes spiral after climbing flight of stairs Has non-smooth tracing with jumps

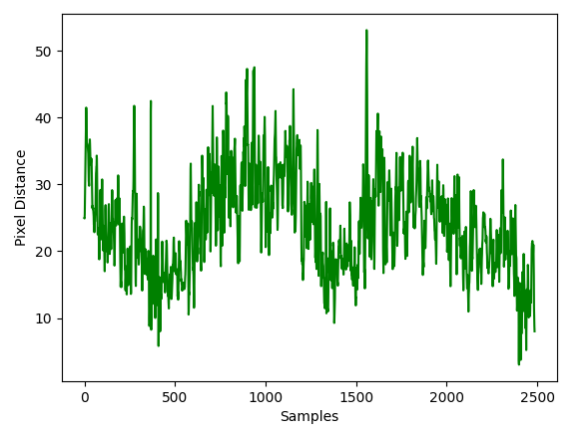

Pixel distance between Archimedes spiral and subject with senile tremor traced line

Power spectral density of subject with senile tremor

Figure 5. Subject with senile tremor - Age 68

This indicates that the two sets of standard deviation values (are from senile tremor and the other set from alcohol induced tremor) seemed to be distinct; in principle this may form a distinctive feature for identification of tremor cause.

\section{B. Tremor Analysis during a task subject to external Stressors.}

In this section we analyse the Archimedes spiral drawn by the subjects after they have been subjected to external stressors. The stressors themselves vary from mild to strong. The mild stressor involved the subjects running up and down a single flight of stairs (between two floors) once. The strongest stressor they were subject to involved running up and down the same flight of stairs three times. After being subjected to the stressors the subjects are required to trace out the Archimedes spiral while wearing the sensor glove with the embedded IMUs and LED. Mean, Median, Variance and Standard deviation value of the pixel distance is calculated for the subjects. 


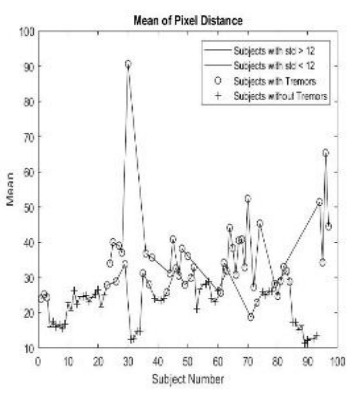

Figure 6. Mean of pixel distance of tremor subjects is greater than healthy subjects

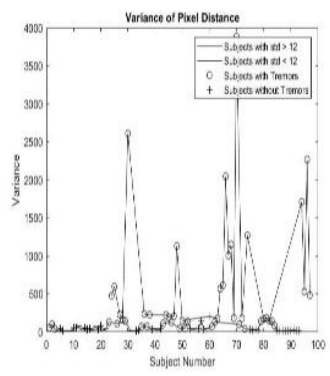

Figure 8. Variance of pixel distance of tremor subjects is greater than healthy subjects

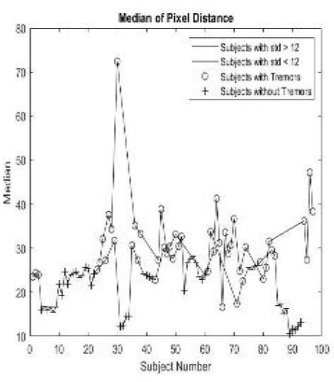

Figure 7. Median of pixel distance of tremor subjects i greater than healthy subject:

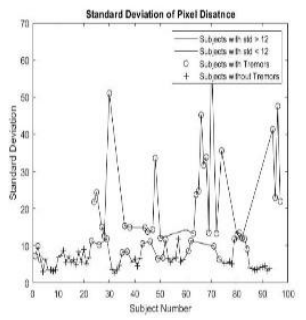

Figure 9. Standard deviation of pixel distance of tremor subjects is greater than healthy subjects

Figures $6,7,8$ and 9 show the mean, median, variance and standard deviation error between the spiral traced by the subjects and the correct Archimedes spiral. As seen from Figure 6, mean of pixel distance for all tremor subjects is closer to 39 whereas mean of pixel distance for healthy subjects is closer to 22. Standard deviation of pixel distance for all tremor subjects is closer to 24 whereas standard deviation of pixel distance for healthy subjects is closer to 7 , as seen from Figure 9.

\section{Experimentation and Analysis}

Our experimentation involved study of 25 subjects. The analysis from these subjects is taken forward to construct an efficient tremor - non tremor classifier model.

\section{A. Dataset}

In this study, both video and accelerometer data of 25 subjects were collected. Out of 25 subjects, 14 subjects had tremor.

\begin{tabular}{|l|l|l|}
\hline \multicolumn{3}{|c|}{ Total Subjects (25) } \\
\hline \multicolumn{2}{|c|}{ Tremor Subjects (14) } & $\begin{array}{c}\text { Healthy } \\
\text { Subjects (11) }\end{array}$ \\
\hline $\begin{array}{l}\text { Senile } \\
\text { Tremor (5) }\end{array}$ & $\begin{array}{l}\text { Alcohol Induced } \\
\text { Tremor (9) }\end{array}$ \\
\hline
\end{tabular}

For our first analysis, five sensors were employed. Six features for each five sensors were extracted i.e thirty features. Statistical features like mean, median, variance, standard

deviation, skewness and kurtosis are derived from absolute value of acceleration (AVA) signal. The dataset generated with all subjects, using accelerometer data is denoted as DS-ACC.

The second analysis was conducted by asking the subjects to trace the Archimedes spiral. The reference spiral was projected on the wall and the subjects were asked to trace using the laser diode, mounted on the glove of the index finger. The video recording of the same was captured. The analysis of the video aimed at the deriving the Pixel distance between reference and traced spirals is calculated. The pixel distance error indicated the severity of the tremor within the subjects. Six features namely, mean, median, variance, standard deviation, skewness and kurtosis features are derived from pixel error signal. The dataset generated with all subjects, using the pixel distances is denoted as DS-ASV.

Dataset1 (DS-ACC) comprises a total of thirty features (derived from accelerometer experiment). Dataset2 (DS-ASV) consists of six features (derived from the Archimedes model). Dataset3 (DS-ACC-ASV) is a compilation of attributes from dataset1 and dataset2.

\section{B. Classifier Model}

Automatic tremor classifier based on the temporal features was developed using machine-learning algorithms: Logistic regression, Naive Bayes, Random Forest, $\mathrm{K}$ nearest neighbor $(\mathrm{KNN})$, Decision Tree (DT) and Support Vector Machine (SVM) algorithms were employed for estimating the presence of tremors. The data were subjected to 5 cross validations. The results were averaged and tested against the above reference models. The kernels employed with SVM were linear. Radial basis function (RBF) and sigmoid. All offline analyses were carried out using Python and Machine learning libraries. The performance measures Accuracy, precision, recall and F Score were used for analysis.

\section{Test Bed.}

A three-step experimental setup is built for coming up with an efficient identification of tremor subjects.

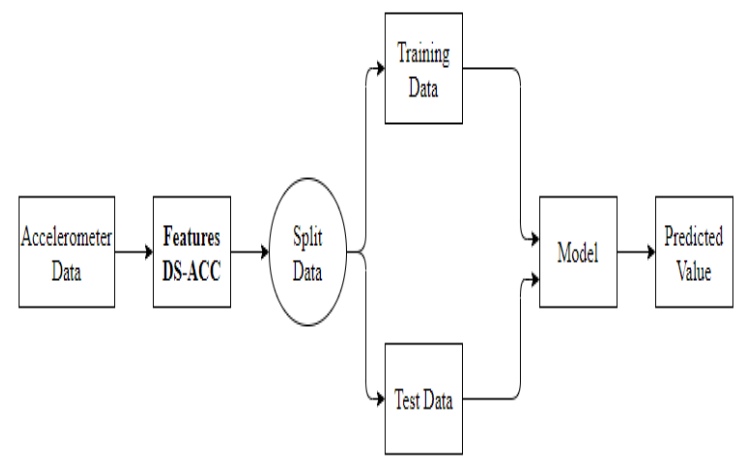

Figure 10. Accelerometer data (DS-ACC) classifier model

Our first setup is as shown in Figure 10. In this study, the model was trained for the features of the accelerometer data 
(DS-ACC). The classifier model was trained and tested against the accelerometer data. The processed feature values were retrieved from raw data from five sensors attached to each finger of the subject's hand. Figure 11 shows the second setup for constructing the classifier model. Features extracted from Archimedes spiral (DS-ASV) were taken for analysis. The model was constructed on six feature datasets.

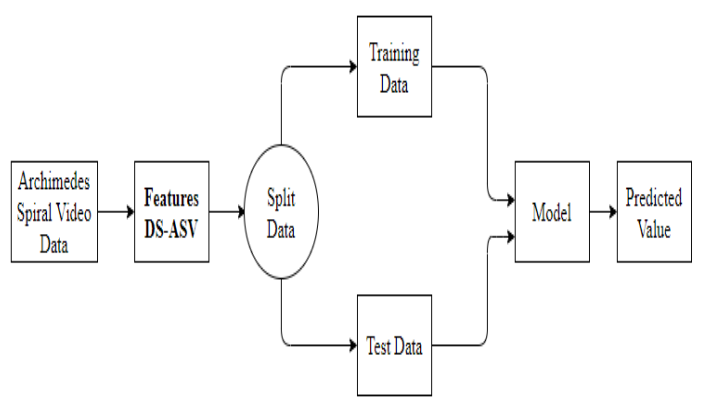

Figure 11. Spiral video data (DS-ASV) classifier model

Our last setup is to combine all the features derived from the experimental process involving the features extracted from accelerometer and Archimedes spiral video (DS-ACC-AVS). The proposed classifier model is as shown in Figure 12.

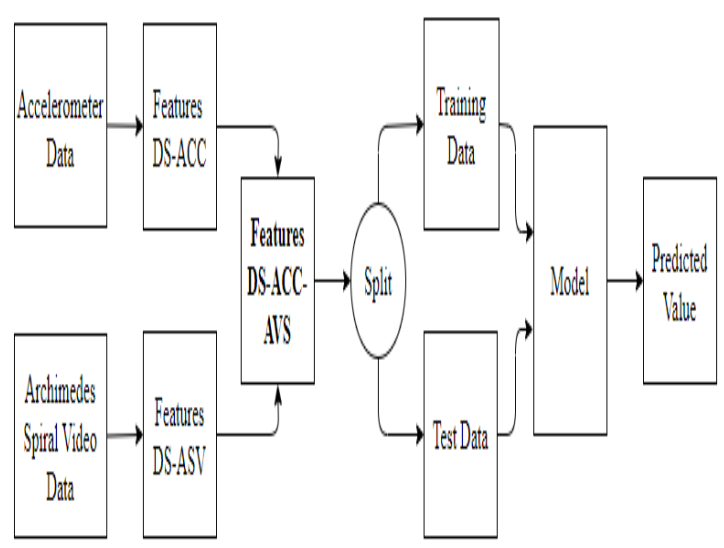

Figure 12. DS-ACC-ASV data classifier Model

\section{Discussion and Results.}

The performance of various classifier models is recorded and analysed. The evaluation metrics to measure the efficiency of the model are Accuracy, Precision, Recall, F1 score.

Accuracy score is plotted indicating the classifier performance for tremor detection as shown in Figure 13.

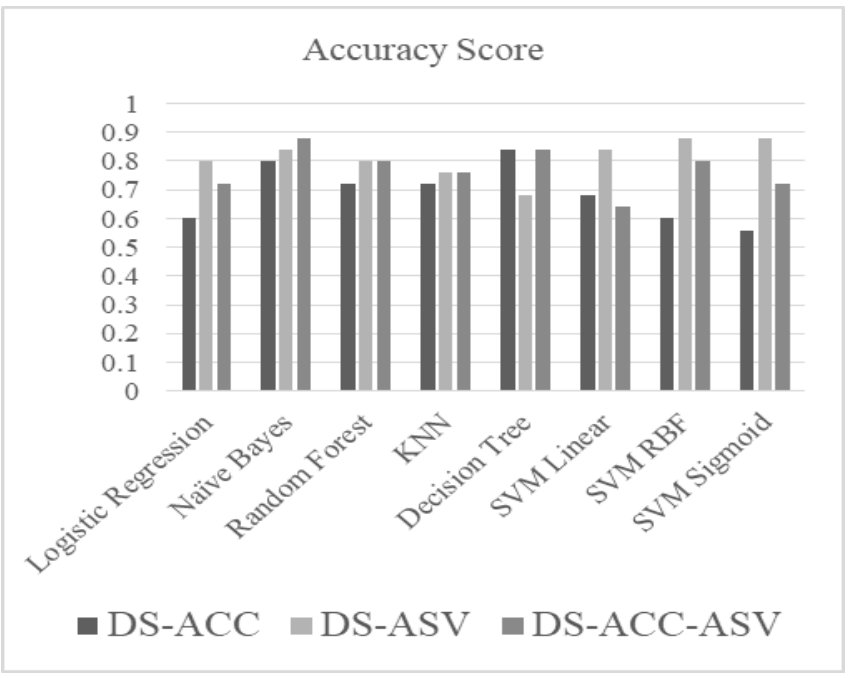

Figure 13. Accuracy score of classifiers for DS-ACC, DSASV, DS-ACC-ASV

Accuracy scores as shown in Figure 13 indicates that the feature data set of Archimedes spiral video (DS-ASV) is most efficient. On comparison of accuracy values SVM is the best classifier. Feature set of DS-ACC-ASV also have given comparable performance in classifying Tremor and Non tremor subjects.

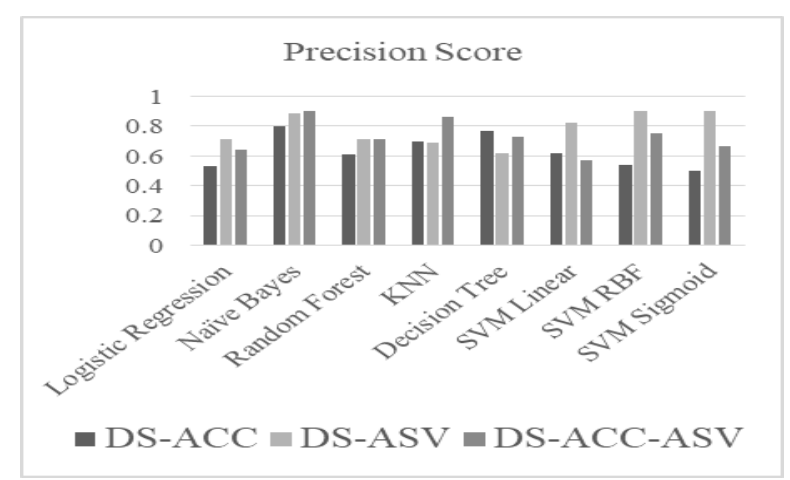

Figure 14. Precision score of classifiers for DS-ACC, DSAVS, DS-ACC-AVS

Precision scores as shown in Figure 14 indicate the goodness of classifier against the tremor subjects. Commendable results are obtained from SVM classifiers. Good performance was also obtained from DS-ASV to identify the positive predictions. 


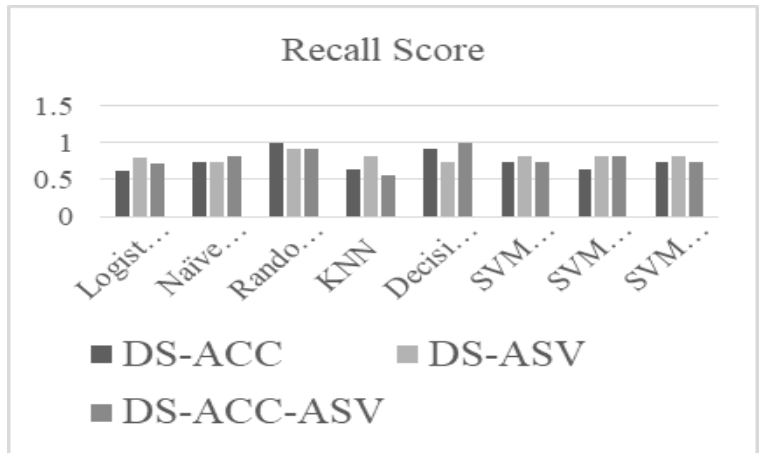

Figure 15. Recall score of classifiers for DS-ACC, DS-AVS, DS-ACC-AVS

As seen from Figure 15, the feature sets of DS-ACC-ASV significantly outperformed for Random Forest, Decision Tree, SVM RBF classifiers to identify Tremor subjects.

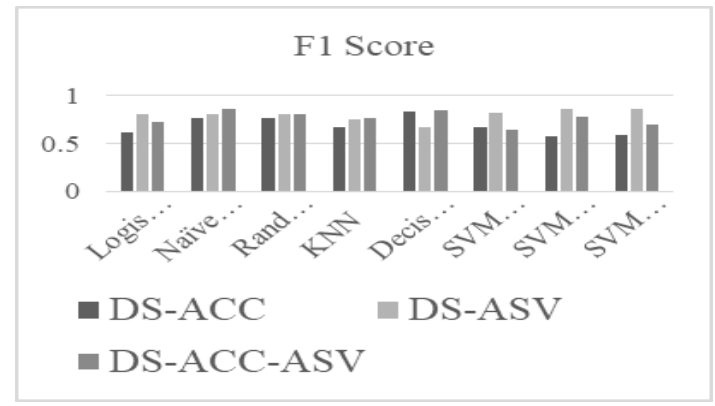

Figure 16. F1 score of classifiers for DS-ACC, DS-AVS, DSACC-AVS

As seen from bar graph (Figure 16), F1score improves the overall performance of model. Figure 17, summarises the overall performance of the classifier model. Accuracy and error rate are the universally accepted standard metrics for estimating the performance of classification models. Accuracy score result for DS-ACC-ASV shows that Naïve Bayes, Random Forest, Decision Tree and SVM classifiers predicted tremor and non-tremor subjects more accurately.

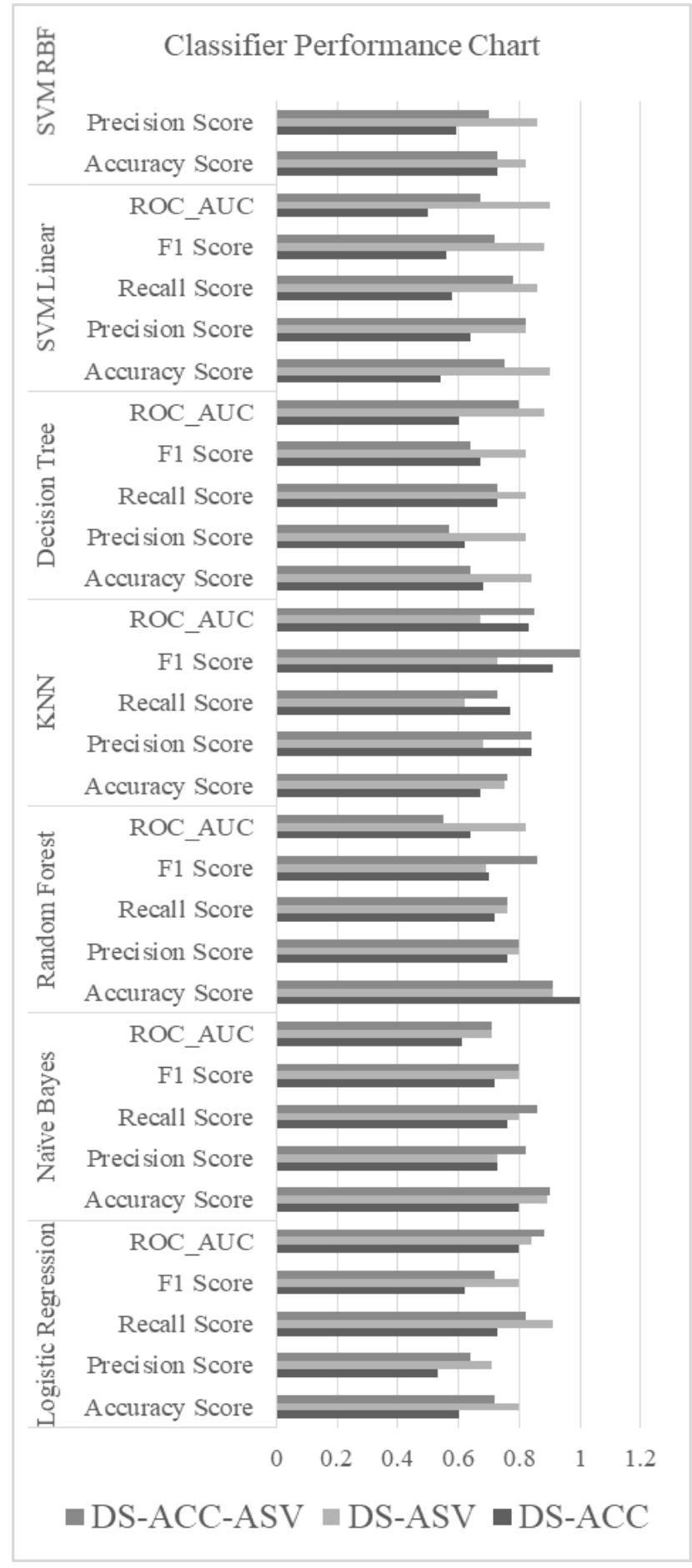

Figure 17. Classifier performance chart

\section{CONCLUSION}

In this work, a novel low-cost wearable sensing device was designed to analyze tremor signals using microcontroller, IMUs and an optical sensor. A novel spirogram analysis was performed using a single camera, a laser diode and our instrumented glove. Unlike in standard clinical approaches where touch sensitive digitized screens are used for handwriting analysis, our proposed system, showed capability of performing tremor analysis for various functional tasks - 
apart from just handwriting analysis. Further, subjects were given external stressors (physical stressors) before having to draw a spirogram. The presence of tremors was detected by the development of an automatic tremor detection system using machine-learning approaches. The use of Naïve Bayes, Random Forest, Decision Tree and SVM were explored with three kernels for the selected features. Accuracy and error rate are the universally accepted standard metrics for estimating the performance of classification models. Accuracy score result for DS-ACC-ASV shows that Naïve Bayes, Random Forest, Decision Tree and SVM classifiers predicted tremor and nontremor subjects more accurately. The principal advantage of our approach is to provide reliable tremor prediction using a wearable sensing device in the presence of external stressors. To conclude, detecting tremors through analysis of patterns drawn by the subjects was found to be a promising and convenient method of estimation. The process was reliable and stable under various physical stress factors such a climbing a flight of stairs. As an future scope of the work it can be noticed that other possible conditions like various age and gender can be considered. Further it can be carried out through the different geographical locations.

\section{ACKNOWLEDGMENT}

"A part of this work was supported by a BIRAC grant Ref. No. BT/AIR094S/PACE-19/19". The authors acknowledge Dr. Triveni, Dr. Asha, nurses and the subjects from primary health care centre Aluru.

\section{References}

[1] Deuschl, G., Bain, P., Brin, M., \& Ad Hoc Scientific Committee. (1998). Consensus statement of the movement disorder society on tremor. Movement disorders, 13(S3), 2-23.

[2] Savitt, J. M., Dawson, V. L., \& Dawson, T. M. (2006). Diagnosis and treatment of Parkinson disease: molecules to medicine. The Journal of clinical investigation, 116(7), 1744-1754.

[3] Jankovic, J. (2008). Parkinson's disease: clinical features and diagnosis. Journal of neurology, neurosurgery \& psychiatry, 79(4), 368-376.

[4] Pullman, S. L. (1998). Spiral analysis: a new technique for measuring tremor with a digitizing tablet. Movement disorders, 13(S3), 85-89.

[5] Hess, C. W., \& Pullman, S. L. (2012). Tremor: clinical phenomenology and assessment techniques. Tremor and other hyperkinetic movements.

[6] Saunders- Pullman, R., Derby, C., Stanley, K., Floyd, A., Bressman, S., Lipton, R. B., ... \& Pullman, S. L. (2008). Validity of spiral analysis in early Parkinson's disease. Movement disorders: official journal of the Movement Disorder Society, 23(4), 531-537.

[7] Liu, X., Carroll, C. B., Wang, S. Y., Zajicek, J., \& Bain, P. G. (2005). Quantifying drug-induced dyskinesias in the arms using digitised spiral-drawing tasks. Journal of neuroscience methods, 144(1), 47-52.

[8] Miralles, F., Tarongi, S., \& Espino, A. (2006). Quantification of the drawing of an Archimedes spiral through the analysis of its digitized picture. Journal of neuroscience methods, 152(1-2), 18-31.

[9] Aly, N. M., Playfer, J. R., Smith, S. L., \& Halliday, D. M. (2007). A novel computer-based technique for the assessment of tremor in Parkinson's disease. Age and Ageing, 36(4), 395-399.

[10] Surangsrirat, D., Intarapanich, A., Thanawattano, C., Bhidayasiri, R., Petchrutchatachart, S., \& Anan, C. (2013, April). Tremor assessment using spiral analysis in time-frequency domain. In 2013 Proceedings of IEEE Southeastcon (pp. 1-6).

[11] Deepali Koppad, "Arrhythmia Classification Using Deep Learning: A Review", WSEAS Transactions on Biology and Biomedicine, vol. 18, pp. 96-105, 2021.

[12] Cunningham, L., Nugent, C., Moore, G., Finlay, D., \& Craig, D. (2009, July). Computer-based assessment of bradykinesia, akinesia and rigidity in Parkinson's disease. In International Conference on Smart Homes and Health Telematics (pp. 1-8). Springer, Berlin, Heidelberg.

[13] Wang, M., Wang, B., Zou, J., Zhang, J., \& Nakamura, M. (2011, May). Quantitative evaluation of hand movement in spiral drawing for patients with Parkinson's disease based on modeling in polar coordinate system with varied origin. In The 2011 IEEE/ICME International Conference on Complex Medical Engineering (pp. 169173). IEEE.

[14] Joundi, R. A., Brittain, J. S., Jenkinson, N., Green, A. L., \& Aziz, T. (2011). Rapid tremor frequency assessment with the iPhone accelerometer. Parkinsonism \& related disorders, 17(4), 288-290.

[15] Albert, M. V., Toledo, S., Shapiro, M., \& Koerding, K. (2012). Using mobile phones for activity recognition in Parkinson's patients. Frontiers in neurology, 3, 158.

[16] Surangsrirat, D., \& Thanawattano, C. (2012, March). Android application for spiral analysis in Parkinson's Disease. In 2012 Proceedings of IEEE Southeastcon (pp. 1-6). IEEE.

[17] Alty, J. E., \& Kempster, P. A. (2011). A practical guide to the differential diagnosis of tremor. Postgraduate medical journal, 87(1031), 623-629.

[18] Coelho, M., Marti, M. J., Tolosa, E., Ferreira, J. J., Valldeoriola, F., Rosa, M., \& Sampaio, C. (2010). Latestage Parkinson's disease: the Barcelona and Lisbon cohort. Journal of neurology, 257(9),1524-1532.

[19] Alves, G., Larsen, J. P., Emre, M., Wentzel- Larsen, T., \& Aarsland, D. (2006). Changes in motor subtype and risk for incident dementia in Parkinson's disease. Movement disorders: official journal of the Movement Disorder Society, 21(8), 1123-1130.

[20] Louis, E. D., \& Machado, D. G. (2015). Tremor-related quality of life: a comparison of essential tremor vs. Parkinson's disease patients. Parkinsonism \& related disorders, 21(7), 729-735.

[21] Louis, E. D. (2016). More time with tremor: the experience of essential tremor versus Parkinson's disease patients. Movement disorders clinical practice, 3(1), 3642. 
[22] Jankovic, J., \& Kapadia, A. S. (2001). Functional decline in Parkinson disease. Archives of neurology, 58(10), 1611-1615.

[23] De Rijk, M. D., Launer, L. J., Berger, K., Breteler, M.M., Dartigues, J. F., Baldereschi, M., ... \& Hofman, A. (2000). Prevalence of Parkinson's disease in Europe: A collaborative study of population-based cohorts. Neurologic Diseases in the Elderly Research Group. Neurology, 54(11 Suppl 5), S21-3.

[24] Louis, E. D., \& Ferreira, J. J. (2010). How common is the most common adult movement disorder? Update on the worldwide prevalence of essential tremor. Movement Disorders, 25(5), 534-541.

[25] Crawford III, P. F., \& Zimmerman, E. E. (2011). Differentiation and diagnosis of tremor. American family physician, 83(6), 697-702.

[26] Salarian, A., Russmann, H., Wider, C., Burkhard, P. R., Vingerhoets, F. J., \& Aminian, K. (2007). Quantification of tremor and bradykinesia in Parkinson's disease using a novel ambulatory monitoring system. IEEE Transactions on biomedical engineering, 54(2), 313-322.

[27] Tanzina Rahman Hera, Md. Ashikur Rahman Khan, Nishu Nath, "Early Gestational Diabetes Detection Using Neural Network", WSEAS Transactions on Biology and Biomedicine, vol. 18, pp. 1-17, 2021.

[28] Deuschl, G., Bain, P., Brin, M., \& Ad Hoc Scientific Committee. (1998). Consensus statement of the movement disorder society on tremor. Movement disorders, 13(S3), 2-23.

[29] Sanjay H S, Bhargavi S, Madhuri S, "Auditory Psychophysical Analysis Of Healthy Individuals Based On Audiometry And Absolute Threshold Tests“, International Journal of Engineering and Technology, Vol 7(19), 99-106

(DOI: 10.14419/ijet.v7i1.9.9742)

[30] Mittel, C. S. (2003). Parkinson's disease: Overview and current abstracts.

[31] Hess, C. W., \& Pullman, S. L. (2012). Tremor: clinical phenomenology and assessment techniques. Tremor and other hyperkinetic movements, 2.

[32] Hauser, R. A., Friedlander, J., Zesiewicz, T. A., Adler, C. H., Seeberger, L. C., O'Brien, C. F., ... \& Factor, S. A. (2000). A home diary to assess functional status in patients with Parkinson's disease with motor fluctuations and dyskinesia. Clinical neuropharmacology, 23(2), 7581.

[33] Andrade, A. O., Pereira, A. A., Soares, M. F., de Almeida, G. L. C., Paixão, A. P. S., Fenelon, S. B., \& Dionisio, V. C. (2013). Human tremor: origins, detection and quantification. Practical Applications in Biomedical Engineering.

[34] Panicker, J. N., \& Pal, P. K. (2003). Clinical features, assessment and treatment of essential tremor. Journalassociation of physicians of india, 51, 276-285.

[35] Mario, M., Giuliana, G., Thomas, L., Dario, F., Lana, P., Silvia, C., .. \& Eduardo, R. (2009). Bioinformatic approaches used in modelling human tremor. Current Bioinformatics, 4(2), 154-172.

[36] Palmes, P., Ang, W. T., Widjaja, F., Tan, L. C., \& Au, W. L. (2010). Pattern mining of multichannel sEMG for tremor classification. IEEE transactions on biomedical engineering, 57(12), 2795-2805.

[37] Rissanen, S. M., Kankaanpää, M., Tarvainen, M. P., Meigal, A., Nuutinen, J., Jäkälä, P., ... \& Karjalainen, P. A. (2010, January). Discrimination of EMG and acceleration measurements between patients with Parkinson's disease and healthy persons. In 2010 Annual International Conference of the IEEE Engineering in Medicine and Biology (pp. 4878-4881). IEEE.

[38] Di Pino, G., Formica, D., Melgari, J. M., Taffoni, F., Salomone, G., di Biase, L., ... \& Guglielmelli, E. (2012, June). Neurophysiological bases of tremors and accelerometric parameters analysis. In 2012 4th IEEE RAS \& EMBS International Conference on Biomedical Robotics and Biomechatronics (BioRob) (pp. 18201825). IEEE.

[39] Rigas, G., Tzallas, A. T., Tsipouras, M. G., Bougia, P., Tripoliti, E. E., Baga, D., ... \& Konitsiotis, S. (2012). Assessment of tremor activity in the Parkinson's disease using a set of wearable sensors. IEEE Transactions on Information Technology in Biomedicine, 16(3), 478-487.

[40] Niazmand, K., Kalaras, A., Dai, H., \& Lueth, T. C. (2011, October). Comparison of methods for tremor frequency analysis for patients with parkinson's disease. In 2011 4th International Conference on Biomedical Engineering and Informatics (BMEI) (Vol. 2, pp. 693-697). IEEE.

[41] Pascoal-Faria, P., Patrício, M., Leal, A., Caramelo, F., Freire, A., Januário, C., \& Castelo-Branco, M. (2014). Understanding tremor in rapid upper limb movements using $3 \mathrm{~d}$ accelerometers data. Neuroscience \& Medicine, 5(05), 205.

[42] Thanawattano, C., Pongthornseri, R., Anan, C., Dumnin, S., \& Bhidayasiri, R. (2015). Temporal fluctuations of tremor signals from inertial sensor: a preliminary study in differentiating Parkinson's disease from essential tremor. Biomedical engineering online, 14(1), 1-13.

[43] Ayache, S. S., Al-Ani, T., \& Lefaucheur, J. P. (2014). Distinction between essential and physiological tremor using Hilbert-Huang transform. Neurophysiologie Clinique/Clinical Neurophysiology, 44(2), 203-212.

[44] Šprdlík, O., Hurák, Z., Hoskovcová, M., Ulmanová, O., \& Růžička, E. (2011). Tremor analysis by decomposition of acceleration into gravity and inertial acceleration using inertial measurement unit. Biomedical Signal Processing and Control, 6(3),269-279.

[45] Martinez-Manzanera, O., Roosma, E., Beudel, M., Borgemeester, R. W. K., van Laar, T., \& Maurits, N. M. (2015). A method for automatic and objective scoring of bradykinesia using orientation sensors and classification algorithms. IEEE Transactions on Biomedical Engineering, 63(5), 1016-1024.

[46] Giuberti, M., Ferrari, G., Contin, L., Cimolin, V., Azzaro, C., Albani, G., \& Mauro, A. (2015). Assigning UPDRS scores in the leg agility task of Parkinsonians: Can it be 
done through BSN-based kinematic variables?. IEEE Internet of Things Journal, 2(1), 41-51.

[47] Giuberti, M., Ferrari, G., Contin, L., Cimolin, V., Azzaro, C., Albani, G., \& Mauro, A. (2015). Automatic UPDRS evaluation in the sit-to-stand task of Parkinsonians: Kinematic analysis and comparative outlook on the leg agility task. IEEE journal of biomedical and health informatics, 19(3), 803-814.

[48] Parisi, F., Ferrari, G., Giuberti, M., Contin, L., Cimolin, V., Azzaro, C., ... \& Mauro, A. (2015). Body-sensornetwork-based kinematic characterization and comparative outlook of UPDRS scoring in leg agility, sit-to-stand, and Gait tasks in Parkinson's disease. IEEE journal of biomedical and health informatics, 19(6), 1777-1793.

[49] Parisi, F., Ferrari, G., Giuberti, M., Contin, L., Cimolin, V., Azzaro, C., ... \& Mauro, A. (2016). Inertial BSNbased characterization and automatic UPDRS evaluation of the gait task of Parkinsonians. IEEE Transactions on Affective Computing, 7(3),258-271.

[50] Samà, A., Pérez-López, C., Rodríguez-Martín, D., Català, A., Moreno-Aróstegui, J. M., Cabestany, J., ... \& Rodríguez-Molinero, A. (2017). Estimating bradykinesia severity in Parkinson's disease by analysing gait through a waist-worn sensor. Computers in biology and medicine, 84, 114-123.

[51] Memedi, M., Khan, T., Grenholm, P., Nyholm, D., \& Westin, J. (2013). Automatic and objective assessment of alternating tapping performance in Parkinson's disease. Sensors, 13(12), 16965-16984.

[52] Stamatakis, J., Ambroise, J., Crémers, J., Sharei, H., Delvaux, V., Macq, B., \& Garraux, G. (2013). Finger tapping clinimetric score prediction in Parkinson's disease using low-cost accelerometers. Computational intelligence and neuroscience, 2013.

[53] Giuffrida, J. P., Riley, D. E., Maddux, B. N., \& Heldman, D. A. (2009). Clinically deployable Kinesia ${ }^{\mathrm{TM}}$ technology for automated tremor assessment. Movement disorders: official journal of the Movement Disorder Society, 24(5), 723-730.

[54] Pan, D., Dhall, R., Lieberman, A., \& Petitti, D. B. (2015). A mobile cloud-based Parkinson's disease assessment system for home-based monitoring. JMIR mHealth and uHealth, 3(1), e3956.

[55] Dai, H., Zhang, P., \& Lueth, T. C. (2015). Quantitative assessment of parkinsonian tremor based on an inertial measurement unit. Sensors, 15(10), 25055-25071.

[56] Jeon, H., Lee, W., Park, H., Lee, H. J., Kim, S. K., Kim, H. B., ... \& Park, K. S. (2017). Automatic classification of tremor severity in Parkinson's disease using a wearable device. Sensors, 17(9), 2067.

[57] Anouti, A., \& Koller, W. C. (1995). Tremor disorders. Diagnosis and management. Western journal of medicine, 162(6), 510.

[58] Mahendra, S.J., Talasila, V., Dutt, A.G (2021). A Wiener Model Based Closed Loop FES for Positional Control During Wrist Flexion, International Journal of Circuits,
Systems and Signal Processing, (Vol. 15, pp. 829-835)), 2021.

[59] Vanitha K M., Viswanath Talasila,, Narasimha Prasad L V., A wearable system to analyze tremors in the presence of external stressors, International Journal of Biomedical Engineering and Technology, Inderscience Publishers.

\section{Creative Commons Attribution License $\mathbf{4 . 0}$ (Attribution 4.0 International, CC BY 4.0)}

This article is published under the terms of the Creative Commons Attribution License 4.0

https://creativecommons.org/licenses/by/4.0/deed.en_US 